\title{
Femoral nerve dimensions at the inguinal ligament and inguinal crease levels: implications for femoral nerve block
}

\author{
OYEDUN, O. S. ${ }^{*}$, RUKEWE, A. ${ }^{2}$ and FATIREGUN, A. ${ }^{3}$ \\ ${ }^{1}$ Gross Anatomy Lab, Department of Anatomy, Faculty of Basic Medical Sciences, \\ University of Ibadan, +234 Ibadan, Oyo State, Nigéria \\ ${ }^{2}$ Anaesthesia Unit, Accident and Emergency Department, University College Hospital, \\ +234 Ibadan, Oyo State, Nigéria \\ ${ }^{3}$ Department of Epidemiology and Medical Statistics, Faculty of Public Health, \\ University of Ibadan, +234 Ibadan, Oyo State, Nigéria \\ *E-mail: oyedundayo@yahoo.com
}

\begin{abstract}
Introduction: Femoral nerve block, when used solely or as a supplement to general anaesthesia, provides anaesthesia and analgesia to the anterior thigh. In spite of its established benefits, femoral nerve block is still underutilized in Nigeria. Our objective was to study the dimensions of femoral nerve at the level of the inguinal ligament and inguinal crease using a cadaveric model; no such data exists in Nigeria. Materials and Methods: Using 7 adult human cadavers ( 6 males and 1 female), the depth and thickness of the femoral nerve were measured at the levels of inguinal ligament and inguinal crease. The spatial relationship of femoral nerve to the surrounding structures was also observed. Result: The study showed a significantly wider thickness and shorter depth of the femoral nerve at the level of inguinal crease relative to inguinal ligament. Conclusion: We concluded that in centers where ultrasound and neurostimulation techniques for femoral nerve block in Nigerians are unavailable, the inguinal crease level where the femoral nerve is more superficial and wider in thickness would be the landmark of choice compared to the inguinal ligament level.
\end{abstract}

Keywords: dimensions, femoral nerve, femoral nerve block, inguinal crease, inguinal ligament.

\section{Introduction}

Femoral nerve block is a basic anaesthetic procedure commonly used in many orthopaedic and plastic reconstructive surgeries. This nerve block technique provides anaesthesia and analgesia to the anterior thigh muscles and extensors of the hip. This block may be a preferred choice to centrineural analgesia due to its superior pain control, lesser side effects, high cost effectiveness, improved recovery and decreased need for post anaesthesia nursing care (PHILIP, 1992; MINGUS, 1995; BRIDENBAUGH, 1983). In spite of these established benefits, femoral nerve block and indeed peripheral nerve blocks are underutilized in many countries (HADZIC, VLOKA, KURODA et al., 1998; RUDKIN and MICALLEF, 2004; RUKEWE and FATIREGUN 2010). Amongst other causes, the underutilization of femoral blocks may be due to the unpredictability of needle- femoral nerve contact at the classical anatomical landmarks. The anatomical sites for femoral nerve block have been studied extensively by a number of investigators (KHOO and BROWN, 1983; VLOKA, HADZIC, DROBNIK et al., 1999; MOORE, 1992; BROWN, 1992). In Nigeria, block anaesthesia using ultrasound and neurostimulation techniques are not readily available in many centres, making it imperative to study anatomical landmark for femoral nerve blocks using a cadaveric model. Our objective was to study the dimensions of femoral nerve at the level of the inguinal ligament and inguinal crease as a step towards determining an effective anatomical site for femoral nerve block. We also set out to study the spatial relationship of structures surrounding the femoral nerve at these anatomical sites.

\section{Materials and Methods}

This descriptive study was conducted on fourteen thighs from seven adult cadavers donated to the Department of Anatomy, University of Ibadan, Nigeria. The cadavers, comprising six males and one female, had been previously embalmed for educational purposes using 10\% formaldehyde and $28 \%$ glycerin solution. The cadavers were positioned supine on the dissecting table with the anterior thigh facing superiorly. The surface marking of the inguinal ligament was identified as a line joining the anterior superior iliac spine and pubic tubercle (Figure 1). The inguinal crease was also identified and marked as a fold of skin lying inferior and parallel to the inguinal ligament intersecting the upper end of the scrotal/labial fold medially. The femoral triangle was exposed by removing the skin and superficial fascia in series. The dissection was deepened and the fascia iliacus opened to expose the femoral nerve. During dissection, special caution was ensured to preserve the fascia lata in order to facilitate precise measurement of the distance of femoral nerve and femoral artery from the fascia lata. The width of the femoral nerve and its distance from the fascia lata were measured to the nearest $\mathrm{mm}$ at the levels of the inguinal ligament and crease using the Vernier caliper. The distance between the femoral artery and fascia lata at the level of inguinal crease and inguinal ligament was also measured. All data were expressed as mean \pm SD for continuous measures and paired t-test was used to analyze the differences in the distances between the relevant structures at the inguinal crease and inguinal ligament levels. All statistical analysis 
were performed using statistical program (SPSS for windows 17.0, SPSS Inc., Chicago, IL, USA).

\section{Results}

Fourteen thighs from seven cadavers were used for this study. The seven cadavers (six males and one female) were of unknown age and average body builds. The femoral nerve width at the level of the inguinal crease (y) was observed to be greater than the width at the level of inguinal ligament $(\mathrm{x})$ (Figure 1). The cross sectional shape of femoral nerve at the level of the inguinal crease was oval while that at the level of the inguinal ligament tended towards round. The femoral nerve descended through the femoral triangle lateral to the femoral artery and was crossed by the Sartorius muscle.

Table 1 shows the dimensions of femoral nerve at the level of the inguinal ligament and inguinal crease. The width of the femoral nerve at the level of inguinal ligament ranged from $3.49-11.80 \mathrm{~mm}$ with a mean value of $7.23 \pm 2.61 \mathrm{~mm}$. The width of the femoral nerve at the level of inguinal crease ranged from $6.20-13.00 \mathrm{~mm}$ with a mean value of $9.40 \pm 2.06 \mathrm{~mm}$.

Femoral nerve was significantly more superficial at the level of inguinal crease than at the inguinal ligament $(8.50 \pm 4.17 \mathrm{~mm}$ vs $13.39 \pm 6.58 \mathrm{~mm} ; \mathrm{p}=0.0001)$.

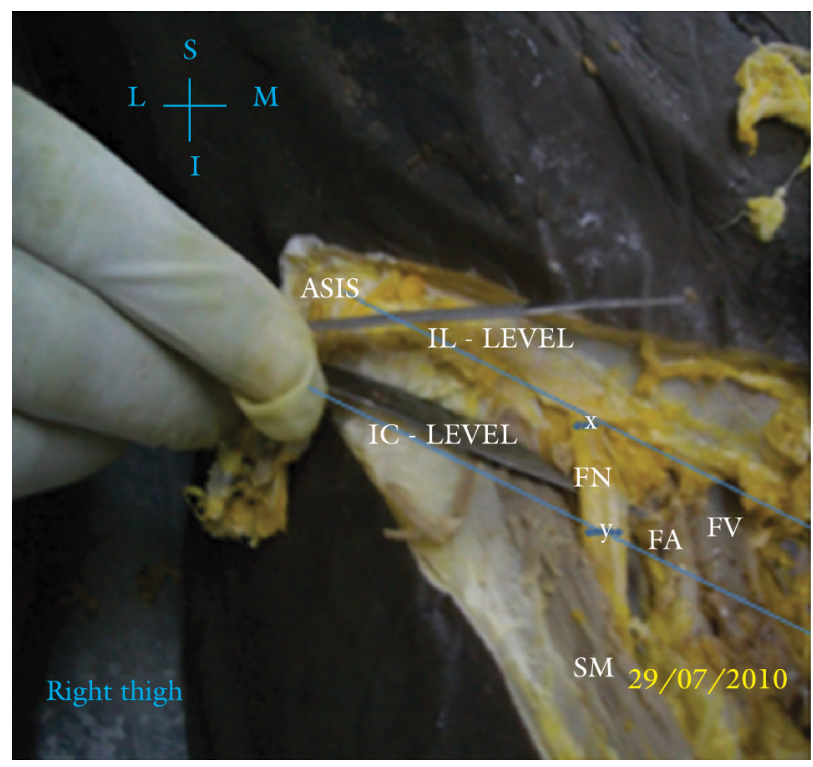

Figure 1. Spatial relationship of the femoral nerve with surrounding structures at IL and IC levels. FN - Femoral nerve. FA - Femoral artery. FV - Femoral vein. SM - Sartorius muscle. IC - Inguinal crease. IL - Inguinal ligament. M - Medial. L - Lateral. S - Superior. I - Inferior. ASIS - Anterior superior iliac spine.

Table 1. Dimensions of the femoral nerve, femoral artery and distances from the fascia lata at the level of IC and IL.

\begin{tabular}{lccc}
\hline FN Width & IC Level & IL Level & P-value \\
\hline FN Width & $9.40 \pm 2.06 \mathrm{~mm}$ & $7.23 \pm 2.61 \mathrm{~mm}$ & 0.009 \\
FN- FL & $8.50 \pm 4.17 \mathrm{~mm}$ & $13.39 \pm 6.58 \mathrm{~mm}$ & 0.0001 \\
FA- FL & $8.98 \pm 5.58 \mathrm{~mm}$ & $12.91 . \pm 6.19 \mathrm{~mm}$ & 0.0001 \\
\hline Key: FN - Femoral nerve FA - Femoral artery FL - Fascia lata. \\
IC - Inguinal crease IL - Inguinal ligament.
\end{tabular}

The mean width of femoral nerve at the level of inguinal crease $(9.40 \pm 2.06 \mathrm{~mm})$ was significantly greater than at the level of inguinal ligament $(7.23 \pm 2.61 \mathrm{~mm} ; \mathrm{p}=0.009)$.

The femoral artery was significantly more superficial at the inguinal crease than at inguinal ligament $(8.98 \pm 5.58 \mathrm{~mm}$ vs 12.91. $\pm .19 \mathrm{~mm} ; \mathrm{p}=0.0001$ ) (Table 1 ).

\section{Discussion}

Our study demonstrated that the femoral nerve is wider in transverse diameter and more superficial in location at the level of the inguinal crease than the inguinal ligament. This finding is in agreement with that of Vloka, Hadzic, Drobnik et al. (1999), who showed that the inguinal crease level was the site with the highest likelihood of block needle-femoral nerve contact. This congruous finding notwithstanding, there are differences in the anatomical measurements between the two studies in all the dimensions; for instance, our femoral nerve width at the inguinal crease versus inguinal ligament levels was 9.40 vs $7.23 \mathrm{~mm}$ while theirs was 14.0 vs $9.8 \mathrm{~mm}$. One explanation could be our use of formalin as the principal fixative as opposed to phenol in theirs.

The anatomical landmark for femoral nerve block has been a subject of debate over the years. Other studies however advocated for an insertion site at the level of inguinal ligament or a few centimeters below (MOORE, 1965). Measurements taken at the level of the inguinal crease and inguinal ligament have been shown to be affected by a number of factors. An ultrasonographic study in healthy volunteers demonstrated an increased femoral nerve - femoral artery distance and reduced skin - femoral nerve distance following passive external rotation of the legs (HSU, LU, CHANG et al., 2007). Mehmood, Coleman, Egan et al., (2010), using MRI study in 10 subjects with fractured neck of femur, reported a significant decrease in femoral nerve - femoral artery distance at the level of inguinal ligament. A later ultrasound study using a larger sample size reported no change in femoral nerve-femoral artery distance in patients with fractured neck of femur (TOWNSLEY, WILES, MOPPETT et al., 2013).

In spite of the easy localization of the femoral nerve at the inguinal crease, needle insertion at this level may result in an increased risk of vascular puncture due to a more superficial position of the femoral artery. Our result showed that the femoral artery was significantly more superficial at the inguinal crease compared with the inguinal ligament level, $\mathrm{P}=0.0001$. The origin and superficial position of lateral circumflex femoral artery relative to femoral nerve has been shown to further increase the risk of vascular puncture at the level of inguinal crease (OREBAUGH, 2006).

Our study is limited by the inevitable anatomical distortion of structures following embalmment especially with the use of formalin as the principal fixative. This distortion was minimized by the inclusion of glycerin in the embalmment fluid which aided retention of tissue water. Also the vascular collapse observed in cadavers is minimal in arteries due to their thicker wall relative to veins.

\section{Conclusion}

We concluded that in centers where ultrasound and neurostimulation techniques for femoral nerve block in Nigerians are unavailable, the inguinal crease level where 
the femoral nerve is more superficial and wider in thickness would be the landmark of choice compared to the inguinal ligament level.

\section{References}

BRIDENBAUGH, LD. Regional anaesthesia for out-patient surgery. A summary of 12 years experience. Canadian Journal of Anaesthesia, 1983, vol. 30, n. 5, p. 548-552. http://dx.doi. org/10.1007/BF03007095

BROWN, DL. Femoral block. Atlas of regional anesthesia. Philadelphia: W. B. Saunders, 1992. p. 89-95.

HADZIC, A., VLOKA, JD., KURODA, MM., KOORN, R. and BIRNBACH, DJ. The use of peripheral nerve blocks in anesthesia practice. A national survey. Regional Anesthesia and Pain Medicine, 1998, vol. 23, n. 1, p. 241-246. PMid:9613533

HSU, HT., LU, IC., CHANG, YL., WANG, FY., KUO, YW., CHIU, SL. and CHU KS. lateral rotation of the lower extremity increases the distance between the femoral nerve and femoral artery: An ultrasonographic study. Kaobsiung Journal of Medical Science, 2007, vol 23, n. 12, p 618-23. http://dx.doi.org/10.1016/ S1607-551X(08)70060-3

KHOO, ST. and BROWN, TCK. Femoral nerve block: anatomical basis for a single injection technique. Anaesthesia Intensive Care, 1983, vol. 11, n. 1, p. 40-42. PMid:6859507

MEHMOOD, S., COLEMAN, M., EGAN, M., CROTTY, J. and HARMOND, D. Study of the anatomical position of the femoral nerve by magnetic resonance imaging in patients with fractured neck of femur: relevance to femoral nerve block. Journal of Clinical Anaesthesia, 2010, vol. 22, n. 2, p. 122-125. PMid:20304354. http://dx.doi.org/10.1016/j.jclinane.2009.05.007

MINGUS, ML. Recovery advantages of regional anaesthesia compared with general anaesthesia. Journal of Clinical Anaesthesia, 1995 , vol. 7, n. 7, p. 628-633. http://dx.doi.org/10.1016/09528180(95)00157-3
MOORE, DC. Block of sciatic and femoral nerve: moore DC edition of regional block. 4th ed. Springfield: Charles C. Thomas, 1965. p. 281-288.

MOORE, DC. Block of the sciatic and femoral nerves. In MOORE, DC. (Ed.). Regional block. Springfield: Charles C. Thomas, 1961. p. 219-231

OREBAUGH, SL. The femoral nerve and its relationship to lateral circumflex femoral artery. Anesthesia Analog, 2006, vol. 102, n. 6, p. 1859-1862. PMid:16717338. http://dx.doi.org/10.1213/01. ane.0000217210.44764.0e

TOWNSLEY, P., WILES, MD., MOPPETT, IK. and EMERY, D. An ultrasound study of the anatomical position of the femoral nerve in patients with a fractured neck of femur. International Journal of Perioperative Ultrasound Application Technology, 2013, vol. 2, n. 1, p. 14-18. http://dx.doi.org/10.5005/jp-journals-10027-1027

PHILIP, BK Regional anaesthesia for ambulatory surgery. Journal of Clinical Anaesthesia, 1992, vol. 4, n. 5, p. 355-358. http://dx.doi. org/10.1016/0952-8180(92)90155-T

RUDKIN, GE. and MICALLEF, TA. Impediments to the use of ankle block in Australia. Anesthesia Intensive Care, 2004, vol. 32, n. 3, p. 364-371.

RUKEWE, A. and FATIREGUN, A. The use of regional anesthesia by anesthesiologists in Nigeria. Anesthesia Analog, 2010, vol. 110, n. 1, p. 243-244. PMid:19933532. http://dx.doi.org/10.1213/ ANE.0b013e3181c0f00e

VLOKA, JD., HADZIC, A., DROBNIK, L., ERNEST, A., REISS, W. and THYS, DM. Anatomical Landmarks for femoral nerve block: a comparison of four needle insertion sites. Anesthesia Analog, 1999, vol. 89, n. 6, p. 1467-1470. PMid:10589630 